\title{
A mechanistic model for internal bone remodeling exhibits different dynamic responses in disuse and overload
}

\author{
Scott J. Hazelwood ${ }^{\mathrm{a}, *}$, R. Bruce Martin ${ }^{\mathrm{a}}$, Mark M. Rashid ${ }^{\mathrm{b}}$, Juan J. Rodrigo ${ }^{\mathrm{c}}$ \\ ${ }^{a}$ Orthopaedic Research Laboratories, University of California, Davis, 4635 Second Avenue, Room 2000, Sacramento, CA 95817, USA \\ ${ }^{\mathrm{b}}$ Department of Civil and Environmental Engineering, University of California, Davis, CA 95616, USA \\ ${ }^{\mathrm{c}}$ Department of Orthopaedic Surgery, University of California Davis Medical Center, ACC Building, Suite 3800, Sacramento, CA 95817, USA
}

\begin{abstract}
Bone is a dynamic tissue which, through the process of bone remodeling in the mature skeleton, renews itself during normal function and adapts to mechanical loads. It is, therefore, important to understand the effect of remodeling on the mechanical function of bone, as well as the effect of the inherent time lag in the remodeling process. In this study, we develop a constitutive model for bone remodeling which includes a number of relevant mechanical and biological processes and use this model to address differences in the remodeling behavior as a volume element of bone is placed in disuse or overload. The remodeling parameters exhibited damped oscillatory behavior as the element was placed in disuse, with the amplitude of the oscillations increasing as the severity of disuse increased. In overload situations, the remodeling parameters exhibited critically sensitive behavior for loads beyond a threshold value. These results bear some correspondence to experimental findings, suggesting that the model may be useful when examining the importance of transient responses for bone in disuse, and for investigating the role fatigue damage removal plays in preventing or causing stress fractures. In addition, the constitutive algorithm is currently being employed in finite element simulations of bone adaptation to predict important features of the internal structure of the normal femur, as well as to study bone diseases and their treatment.
\end{abstract}

Keywords: Bone remodeling; Fatigue damage; Disuse; BMU; Computer simulation

\section{Introduction}

Bone is an exceptional tissue in that most of its volume consists of a calcified, load-bearing interstitial matrix. By virtue of its calcification, bone is twice as dense as soft tissues. Consequently, bone's internal structure must be efficient so as to control skeletal weight while maintaining necessary strength (Currey, 1984). Bone tissue that is insufficiently loaded (i.e., in relative "disuse") is removed by remodeling. The maintenance of relatively high skeletal loads means that bone is also subject to the accumulation of fatigue microdamage. A second function of remodeling is to remove this damage, preventing eventual fatigue failure (Parfitt, 1993). Indeed, there is strong experimental evidence that remodeling is activated by disuse (e.g., Li et al., 1990; Schaffler and $\mathrm{Li}$,
1990) and fatigue microdamage (Bentolila et al., 1998; Burr et al., 1985; Burr and Martin, 1993; Mori and Burr, 1993), as well as chemical factors such as estrogen deficiency.

Remodeling is fundamental to bone biology. It is a two-stage process carried out by teams of cells known as basic multicellular units (BMUs). Resorption of a packet of bone by osteoclasts is followed by refilling of the resorption cavity by osteoblasts. This sequence typically requires 3-4 months to complete at each locus, and the resorption and refilling cavities, while individually small, may collectively add substantial temporary porosity or "remodeling space" to the bone. If the purpose of elevated remodeling is to remove bone mass, the remodeling space is inconsequential, but if the goal is damage removal or tissue rearrangement, remodeling's porosity can weaken the bone structure. It is therefore essential that remodeling be understood, not simply as a fundamental biological process, but in the context of bone's load-bearing function. 
It is equally important that bone's mechanical adaptability and resistance to fatigue be understood in the context of remodeling. Many investigators have used computational models to test hypotheses for the mathematical laws governing bone's mechanical adaptation. Simulations have usually assumed that the functional stimulus for adaptation is stress, strain energy, or a related factor such as damage (Beaupré et al., 1990a,b; Carter et al., 1989; Hart et al., 1984; Huiskes et al., 1987; Mullender and Huiskes, 1995; Prendergast and Taylor, 1994; Turner et al., 1997; Weinans et al., 1992). It is significant that most models have been biologically phenomenologic rather than mechanistic. We believe more mechanistic models are needed for two reasons. First they offer more opportunities for validation because, while they must contain remodeling variables that are initially unknown, these variables will often be experimentally measurable. Second, they are likely to reveal effects and relationships missed by less detailed models. Of particular interest are the effect of remodeling space porosity on continuing mechanical function, and the effect of remodeling's inherent time lag on its homeostatic functions.

Here, we pursue a more complete model of bone remodeling. We previously examined the local behavior of a biologically mechanistic model for fatigue damageactivated remodeling (Martin, 1995), but that model did not include the disuse portion of remodeling's function. Ultimately, the more complete system, in which remodeling is activated when loads are either so high as to produce damage, or so low as to represent a disuse state, must be studied in a whole bone model. In that situation, local disuse and overload may occur simultaneously in different regions of the same bone, or sequentially in the same region, and the time lags and non-linearities in the system may produce significant biological complexity. This complexity constitutes an important biological problem because it may contribute to the difficulty in predicting such orthopaedic morbidity as hip fractures and implant loosening. Prior to studying this system in a whole bone model, where dynamic effects in neighboring elements may interact, it is important to understand such effects in an isolated, continuum-level, volume element (Fig. 1). Here, we address the question, does the remodeling response to disuse in a single-volume element include significant dynamic effects and how do they compare to those produced by fatigue overload?

\section{Methods}

The model simulates porosity and material property (elastic modulus) changes brought about by internal bone remodeling provoked by disuse and damage (Fig. 2). Porosity changes are calculated each step in

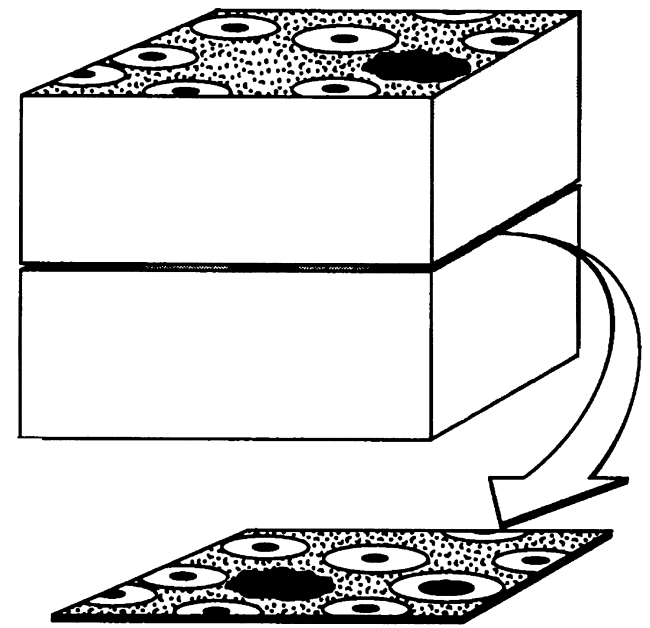

Fig. 1. Example of BMUs in a representative bone volume element. Cortical bone is depicted, but the analysis was also applicable to a volume of trabecular bone. The remodeling analysis was performed for a representative cross-section of the volume as shown schematically here. Bone volumes were assigned linearly elastic and isotropic material properties and a cross-sectional area of $100 \mathrm{~mm}^{2}$ in the analysis. For the purposes of this figure, the schematic representation of the volume has a cross-sectional area of approximately $1 \mathrm{~mm}^{2}$ so individual BMUs may be viewed. Porosity (or void volume fraction) measurements from two-dimensional histologic sections were assumed equivalent to three-dimensional volumetric porosity measurements (Underwood, 1970). In the section shown, one BMU has resorbed bone to its cement line diameter and one BMU is in the refilling stage. (Modified with permission from Martin, 1992.)

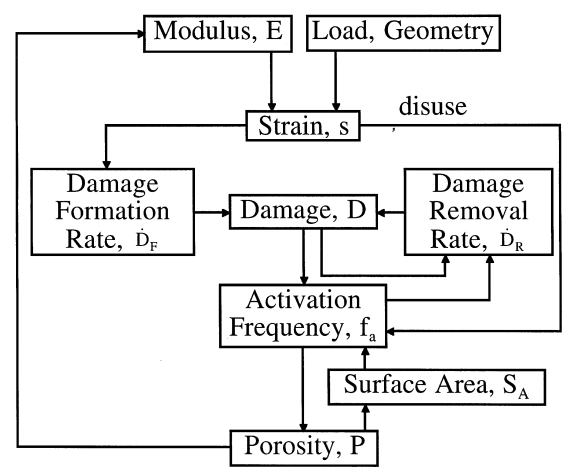

Fig. 2. Schematic representation of the bone remodeling algorithm. Remodeling is activated by disuse (low strain) and damage. Bone porosity is determined from the activation frequency history. Note that this system contains two feedback loops by which remodeling rate affects damage. To the right, remodeling results in damage removal. To the left, increased remodeling results in increased porosity, strain, and damage formation.

time based on temporal and geometric characteristics of remodeling by BMUs in cross-section (Fig. 1). The damage and disuse stimuli are estimated from the strain state of the material and the loading rate. Axial compressive loads are placed on the volume element to simulate loading conditions of different magnitudes, and the material is assumed to be linearly elastic (but with an evolving modulus). Nine state variables are updated at 
each time step of the analysis, and the model parameters are chosen based on experimental observation (Table 1). A sensitivity analysis is performed to identify the influence of each parameter on the resulting remodeling variables. Those parameters having the greatest influence are examined here.

The fatigue damage removal portion of the model follows the development of Martin (1995), but the current model also includes the removal of bone by remodeling when loading falls below a disuse threshold. This disuse portion of the algorithm shares pathways for the effects of remodeling on porosity and elastic modulus with the fatigue damage removal response. However, neither the model's responses to disuse, nor the interactions between the damage and disuse sides of the model, have been previously studied.

\subsection{Relationship between elastic modulus and porosity}

An important component of this model is relating the elastic modulus, $E$, of a bone region to its porosity $(p)$ resulting from resorbing and refilling BMUs. For our study, a relationship between modulus and porosity was obtained by fitting a polynomial to the results of Currey (1988) and Rho et al. (1993), whose data were modified by Turner et al. (1997). By assuming a linear relation- ship between apparent density $(\rho)$ and porosity (for $p=0, \rho=2.0 \mathrm{~g} / \mathrm{cm}^{3}$; for $p=1, \rho=0 \mathrm{~g} / \mathrm{cm}^{3}$ ) we obtained ( $E$ and the coefficients are in units of Mpa)

$$
\begin{aligned}
E= & \left(8.83 \times 10^{5}\right) p^{6}-\left(2.99 \times 10^{6}\right) p^{5}+\left(3.99 \times 10^{6}\right) p^{4} \\
& -\left(2.64 \times 10^{6}\right) p^{3}+\left(9.08 \times 10^{5}\right) p^{2}-\left(1.68 \times 10^{5}\right) p \\
& +\left(2.37 \times 10^{4}\right) .
\end{aligned}
$$

\subsection{Porosity changes}

As proposed by Hart and Davy (1989) and Martin (1985), the rate of change of porosity, $\dot{p}$, was assumed to be a function of the mean bone resorbing $\left(Q_{\mathrm{R}}\right)$ and refilling $\left(Q_{\mathrm{F}}\right)$ rates for each BMU, and the density of resorbing $\left(N_{\mathrm{R}}\right)$ and refilling $\left(N_{\mathrm{F}}\right)$ BMUs/area

$\dot{p}=Q_{\mathrm{R}} N_{\mathrm{R}}-Q_{\mathrm{F}} N_{\mathrm{F}}$.

Here, the resorption $\left(Q_{\mathrm{R}}=A / T_{\mathrm{R}}\right)$ and refilling $\left(Q_{\mathrm{F}}=A / T_{\mathrm{F}}\right)$ rates were assumed to be linear in time, with $A$ representing the area of bone resorbed by each BMU (the area within an osteonal cement line of radius $0.095 \mathrm{~mm}$ as determined by Parfitt, 1983). $T_{\mathrm{R}}$ and $T_{\mathrm{F}}$ are the resorption and refilling periods, respectively. In trabecular bone, BMUs resorb and refill trenches rather than tunnels, but the process is similar and $A$ was

\begin{tabular}{|c|c|c|c|}
\hline & State variable & & \\
\hline E & Elastic modulus (MPa) & & \\
\hline$p$ & Porosity & & \\
\hline$N_{\mathrm{R}}$ & Number of resorbing BMUs (BMUs/mm²) & & \\
\hline$N_{\mathrm{F}}$ & Number of refilling BMUs (BMUs $/ \mathrm{mm}^{2}$ ) & & \\
\hline$f_{\mathrm{a}}$ & BMU activation frequency (BMUs $/ \mathrm{mm}^{2} /$ day) & & \\
\hline$S_{\mathrm{A}}$ & Normalized specific surface area & & \\
\hline$D$ & Damage $\left(\mathrm{mm} / \mathrm{mm}^{2}\right)$ & & \\
\hline$s$ & Strain, $\mu \varepsilon$ & & \\
\hline \multirow[t]{2}{*}{$\Phi$} & Mechanical stimulus (cpd) & & \\
\hline & Constant & Nominal value & Values examined in this study \\
\hline$A$ & Cross-sectional area of each BMU $\left(\mathrm{mm}^{2}\right)$ & $2.84 \times 10^{-2}$ & \\
\hline$T_{\mathrm{R}}$ & Resorption period (days) & 24 & $18-30$ \\
\hline$T_{\mathrm{I}}$ & Reversal period (days) & 8 & \\
\hline$T_{\mathrm{F}}$ & Refilling period (days) & 64 & \\
\hline$k_{\mathrm{D}}$ & Damage rate coefficient $\left(\mathrm{mm} / \mathrm{mm}^{2}\right)$ & $1.85 \times 10^{5}$ & \\
\hline$n$ & Loading conditions & 1 & \\
\hline$q$ & Damage rate exponent & 4 & $1-8$ \\
\hline$R_{\mathrm{L}}$ & Loading rate $(\mathrm{cpd})$ & 3000 & \\
\hline$F_{\mathrm{s}}$ & Damage removal specificity factor & 5 & \\
\hline$D_{0}$ & Initial damage $\left(\mathrm{mm} / \mathrm{mm}^{2}\right)$ & 0.0366 & \\
\hline$f_{\mathrm{a} 0}$ & Initial BMU activation frequency (BMUs/ $\mathrm{mm}^{2} /$ day) & 0.00670 & \\
\hline$\Phi_{0}$ & Initial mechanical stimulus (cpd) & $1.875 \times 10^{-10}$ & \\
\hline$f_{\mathrm{a}(\max )}$ & Maximum BMU activation frequency (BMUs $/ \mathrm{mm}^{2} /$ day) & 0.50 & $0.50-2.50$ \\
\hline$k_{\mathrm{b}}$ & Activation frequency dose-response coefficient $\left(\mathrm{cpd}^{-1}\right)$ & $6.5 \times 10^{10}$ & \\
\hline$k_{\mathrm{c}}$ & Activation frequency dose-response coefficient (cpd) & $9.4 \times 10^{-11}$ & \\
\hline$k_{\mathrm{r}}$ & Activation frequency dose-response coefficient & -1.6 & \\
\hline
\end{tabular}

Table 1

Model state variables and constants 
assumed to be the same for both cortical and trabecular bone.

The populations $N_{\mathrm{R}}$ and $N_{\mathrm{F}}$ were found by integrating over the appropriate resorption $\left(T_{\mathrm{R}}\right)$, reversal $\left(T_{\mathrm{I}}\right)$, and refilling $\left(T_{\mathrm{F}}\right)$ time intervals of the BMU activation frequency $\left(f_{\mathrm{a}}\right)$ history. For a histologic section (Fig. 1), $T_{\mathrm{R}}$ is defined as the period from the moment the osteoclasts of a resorbing BMU enter the section to the time that resorption by these cells ceases in the section. The reversal or inactive period, $T_{\mathrm{I}}$, follows and is the transition from osteoclastic to osteoblastic activity. Osteoblasts of the BMU then form bone in the section during the refilling period, $T_{\mathrm{F}} . N_{\mathrm{R}}$ was found by integrating $f_{\mathrm{a}}$ from time $t-T_{\mathrm{R}}$ to $t$, where $t$ is the present time

$N_{\mathrm{R}}=\int_{t-T_{\mathrm{R}}}^{t} f_{\mathrm{a}}\left(t^{\prime}\right) \mathrm{d} t^{\prime}$

and $N_{\mathrm{F}}$ was found by integrating over the refilling period [time $t-\left(T_{\mathrm{R}}+T_{\mathrm{I}}+T_{\mathrm{F}}\right)$ to $t-\left(T_{\mathrm{R}}+T_{\mathrm{I}}\right)$ ]

$N_{\mathrm{F}}=\int_{t-\left(T_{\mathrm{R}}+T_{\mathrm{I}}+T_{\mathrm{F}}\right)}^{t-\left(T_{\mathrm{R}}+T_{\mathrm{I}}\right)} f_{\mathrm{a}}\left(t^{\prime}\right) \mathrm{d} t^{\prime}$.

Nominal values of $T_{\mathrm{R}}=24$ days, $T_{\mathrm{I}}=8$ days, and $T_{\mathrm{F}}=$ 64 days were calculated from several histomorphometric studies and used in this analysis (Birkenhäger-Frenkel and Birkenhäger, 1987; Brockstedt et al., 1993, 1996; Eriksen, 1986; Frost, 1969; Mellibovsky et al., 1996; Parfitt, 1983, 1994; Pirok et al., 1966; Sarnsethsiri et al., 1971; Steiniche et al., 1994; Stout and Lueck, 1995). The effects of increasing or decreasing the resorption period were examined also by performing the analysis with $T_{\mathrm{R}}=18$ and 30 days.

\subsection{BMU activation frequency}

The BMU activation frequency, $f_{\mathrm{a}}$ (BMUs/area/time), was assumed to be a function of disuse as well as of the existing state of damage. Also, because BMUs must start on a bone surface, $f_{\mathrm{a}}$ was taken to be a function of the internal surface area of the bone region. Specific surface area (internal surface area per unit volume, $S_{\mathrm{A}}$ ) was determined from porosity using an empirical relationship (Martin, 1984), normalized to values between 0 and 1 . Allowance was thus made for the greater potential for remodeling offered by large surface areas within bone by letting

$f_{\mathrm{a}}=\left(f_{\mathrm{a}(\text { disuse })}+f_{\mathrm{a}(\text { damage })}\right) S_{\mathrm{A}}$,

where $f_{\mathrm{a} \text { (disuse) }}$ and $f_{\mathrm{a} \text { (damage) }}$ represent contributions to $f_{\mathrm{a}}$ from disuse and damage, respectively.

\subsection{Rate of fatigue damage accretion}

Damage $(D)$ is defined here as total crack length per section area of bone, although other definitions of damage per unit area (e.g., microcrack density) could be used. Following the development of Martin (1995), the fatigue damage accretion rate is

$\dot{D}=\dot{D}_{\mathrm{F}}-\dot{D}_{\mathrm{R}}$,

where $\dot{D}_{\mathrm{F}}$ and $\dot{D}_{\mathrm{R}}$ represent the fatigue damage formation and removal rates, respectively. $\dot{D}_{\mathrm{F}}$ was assumed to be proportional to the product of the strain range $(s$, microstrain, $\mu \varepsilon)$ raised to a power and the loading rate $\left[R_{\mathrm{L}}\right.$, cycles per unit time], summed over $n$ discrete loading conditions (Caler and Carter, 1989; Carter et al., 1987; Martin, 1992)

$\dot{D}_{\mathrm{F}}=k_{\mathrm{D}} \sum_{i=1}^{n} s_{i}^{q} R_{\mathrm{Li}}=k_{\mathrm{D}} \Phi$.

Here, $\Phi$ is defined as the mechanical stimulus and $k_{\mathrm{D}}$ is a damage rate coefficient. For the sake of simplicity, we assumed that the strain, $s$, was the principal compressive strain and that it returns to zero at the end of each load cycle, so that strain range and peak strain are synonymous. The value for the exponent $q$ was set at a nominal value of 4 based on the results of Whalen and Carter (1988). Values of $q=1,6$, and 8 were also examined in this study. For the purposes of this paper, cyclic loading of only a single magnitude was applied, so that $n=1$. The loading rate, $R_{\mathrm{L}}$, was assumed to be 3000 cycles per day (cpd).

Assuming that BMUs and damage are randomly distributed in the bone, the damage removal rate is $D f_{\mathrm{a}} A$. However, it was assumed that damage initiates BMU activation (Bentolila et al., 1998; Burr et al., 1985; Burr and Martin, 1993; Mori and Burr, 1993), so that the efficiency of damage removal is greater than for random remodeling. To allow for this, a damage removal specificity factor, $F_{\mathrm{s}}$, was included in the equation for the damage removal rate

$\dot{D}_{\mathrm{R}}=D f_{\mathrm{a}} A F_{\mathrm{s}}$.

As in Martin (1995), $F_{\mathrm{S}}$ was set to 5 based on the frequency with which microcracks were associated with new resorption cavities in the experiments of Burr and coworkers (Burr et al., 1985; Burr and Martin, 1993; Mori and Burr, 1993). In a state of equilibrium, $\dot{D}_{\mathrm{F}}=$ $\dot{D}_{\mathrm{R}}$. This provides an estimate of the damage rate coefficient $k_{\mathrm{D}}$ :

$k_{\mathrm{D}}=D_{0} f_{\mathrm{a} 0} A F_{\mathrm{s}} / \Phi_{0}$,

where the subscript 0 indicates the initial, equilibrium values assigned at the start of the simulation.

Using an average crack length of $0.088 \mathrm{~mm}$ (Burr and Martin, 1993) and the average crack density for a 40 year old person from Schaffler et al. (1995), $D_{0}$ was set at $0.0366 \mathrm{~mm} / \mathrm{mm}^{2}$. An initial activation frequency, $f_{\mathrm{a} 0}=0.00670 \mathrm{BMUs} / \mathrm{mm}^{2} /$ day was obtained from averaging several studies of cortical bone (BirkenhägerFrenkel and Birkenhäger, 1987; Brockstedt et al., 1993, 
1996; Frost, 1969; Parfitt, 1983, 1994; Pirok et al., 1966; Sarnsethsiri et al., 1971; Stout and Lueck, 1995). The initial mechanical stimulus $\left(\Phi_{0}\right)$ was estimated from cyclic strain levels necessary to "maintain" cortical bone mass in equilibrium (Lanyon et al., 1975; Rubin and Lanyon, 1981, 1983, 1984). A person walking $4.5 \mathrm{~km} /$ day with a modest $1.5 \mathrm{~m}$ stride (i.e. 2 steps) experiences about $3000 \mathrm{cpd}$ (or roughly 1 million cycles per year) of lower extremity loading. Assuming $R_{\mathrm{L}}=3000 \mathrm{cpd}$ to be typical, the results of Beaupré et al. (1990b) indicate that an equivalent cyclic strain of approximately $500 \mu \varepsilon$ would constitute an equilibrium condition for cortical bone, with an initial mechanical stimulus of $\Phi_{0}=1.875 \times 10^{-10} \mathrm{cpd}$. Substituting these values into Eq. (9) yielded $k_{\mathrm{D}}=1.85 \times 10^{5} \mathrm{~mm} / \mathrm{mm}^{2}$.

\subsection{Disuse}

To formulate the BMU response to disuse, we adopted the "daily stress stimulus" approach of Carter and coworkers (1987). For this study, disuse was defined as stimulus values $\Phi$ below the equilibrium stimulus $\Phi_{0}$. Thus, we use $\Phi$ both to calculate the damage formation rate $\left(\dot{D}_{\mathrm{F}}=k_{\mathrm{D}} \Phi\right)$ and to quantify disuse as $\Phi_{0}-\Phi$, where $\Phi_{0}$ is the initial, equilibrium value. (We do this for convenience; we do not assume that the actual mechanism for remodeling activation is the same for disuse and damage.) Also, when in disuse $\left(\Phi<\Phi_{0}\right)$, the refilling rate in Eq. (2) was determined by setting the area of bone formed to $A\left[0.5+0.5\left(\Phi / \Phi_{0}\right)\right]$ to account for reduced refilling on bone surfaces in disuse states (Frost, 1998).

\subsection{BMU activation frequency response to disuse and damage}

The relationships between the BMU activation frequency $\left(f_{\mathrm{a}}\right)$ and both disuse (Fig. 3a) and damage (Fig. 3b) were assumed to be sigmoidal, similar to responses found in pharmacological applications. The coefficients in these functions were selected to fit the curves within known experimental data ranges. For disuse, we assumed that

$f_{\mathrm{a}(\text { disuse })}=\frac{f_{\mathrm{a}(\max )}}{1+\mathrm{e}^{k_{\mathrm{b}}\left(\Phi-k_{\mathrm{c}}\right)}} \quad$ for $\Phi<\Phi_{0}$,

where $k_{\mathrm{b}} \quad\left(6.5 \times 10^{10} \mathrm{cpd}^{-1}\right)$ and $k_{\mathrm{c}} \quad\left(9.4 \times 10^{-11}\right.$ $\left.\mathrm{cpd}=\Phi_{0} / 2\right)$ are coefficients which define the slope and the inflection point of the curve, respectively.

The relationship between the BMU activation frequency and damage $(D)$ was similar to that used by Martin (1995)

$f_{\mathrm{a}(\text { damage })}=\frac{\left(f_{\mathrm{a} 0}\right)\left(f_{\mathrm{a}(\max )}\right)}{f_{\mathrm{a} 0}+\left(f_{\mathrm{a}(\max )}-f_{\mathrm{a} 0}\right) \mathrm{e}^{\left[k_{\mathrm{r}}\left(f_{\mathrm{a}(\max }\right)\left(D-D_{0} / D_{0}\right)\right]}}$,

where $D_{0}$ is the initial, equilibrium damage and $k_{\mathrm{r}}=$ -1.6 defines the shape of the curve. The nominal value for the maximum activation frequency, $f_{\mathrm{a}(\max )}=0.50 \mathrm{BMUs} / \mathrm{mm}^{2} /$ day, used here was intentionally chosen to be higher than the largest average activation frequency $\left(0.14 \mathrm{BMUs} / \mathrm{mm}^{2} /\right.$ day $)$ measured by Frost (1969) in normal human cortical bone because it is assumed that such measurements did not approach the upper limit of $f_{\mathrm{a}}$ values. Analyses were also performed with $f_{\mathrm{a}(\max )}$ equal to 1.50 and $2.50 \mathrm{BMUs} /$ $\mathrm{mm}^{2}$ /day to investigate the effects of this parameter on the remodeling variables. The range of values for damage $\left(0-0.5 \mathrm{~mm} / \mathrm{mm}^{2}\right)$ used for Eq. (11) was determined from the results of Schaffler et al. (1995).

\subsection{Numerical implementation}

This constitutive model is fundamentally based on two first-order, nonhomogeneous, nonlinear differential equations [Eqs. (2) and (6)] which govern the evolutionary state variables porosity and damage, respectively. The mechanical stimulus $\Phi$ is regarded as the forcing function. The rate equations [Eqs. (2) and (6)] are clearly coupled. Both involve the BMU activation frequency, $f_{\mathrm{a}}$, which itself is not an independent state descriptor, as it is algebraically related to $\Phi, D$, and $p$ (through $S_{\mathrm{A}}$ ), in Eqs. (5), (10) and (11).

The algorithm was implemented using a simple forward Euler scheme to integrate Eqs. (2) and (6) in
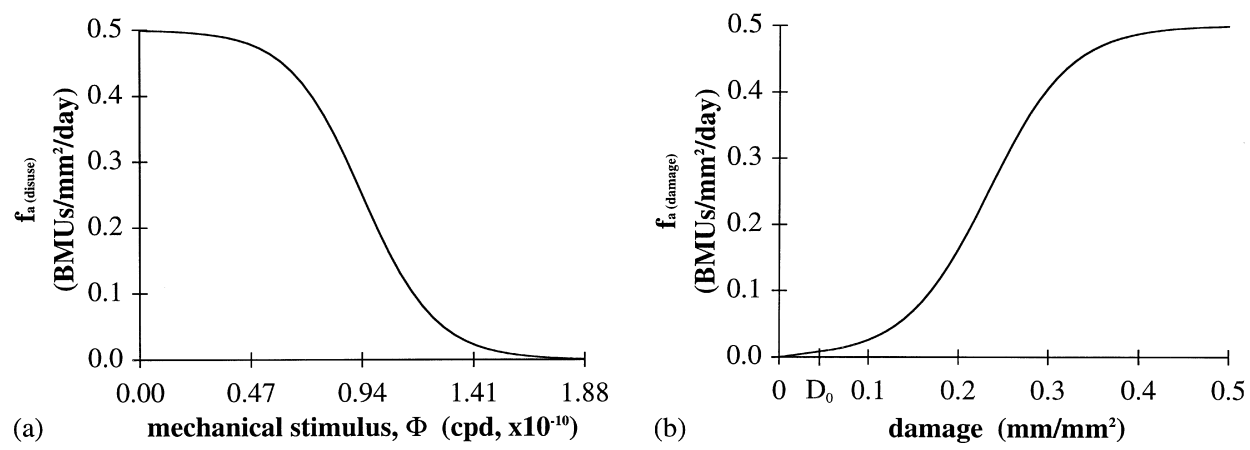

Fig. 3. Proposed relationships between BMU activation frequency and (a) disuse and (b) damage. 
time. Time increments of $0.05,0.1,0.5,1,2,4$, and 8 days were examined. The integrals in Eqs. (3) and (4) were evaluated using the history of the daily average activation frequency. The model was given an initial porosity of $4.43 \%$ because this allows equilibrium between the Haversian canals removed and added by new BMUs (Martin, 1995; Martin et al., 1998). This porosity produced an initial modulus of $17.8 \mathrm{GPa}$ as determined by Eq. (1). Keeping $R_{\mathrm{L}}=3000$ cpd constant, a compressive force of $891.6 \mathrm{~N}$ (producing $500 \mu \varepsilon$ ) provided a mechanical stimulus such that bone resorption and formation were in equilibrium. Remodeling responses to disuse and overload were examined for up to 2000 days by applying lower (10 and $445.8 \mathrm{~N})$ and higher $(1400,1640$, and $1655 \mathrm{~N})$ loads, respectively.

\section{Results}

The model's responses to disuse involved transient oscillatory behavior. Reducing the applied load by $50 \%$ (to $445.8 \mathrm{~N}$ ) and to essentially zero $(10 \mathrm{~N})$ produced new equilibrium porosities of 31.1 and $94.2 \%$, respectively, after 2000 days (Fig. 4, top). However, the BMU activation frequency did not simply rise when the load was reduced, and fall as strain returned to normal, but oscillated sharply (Fig. 4, bottom). These transient
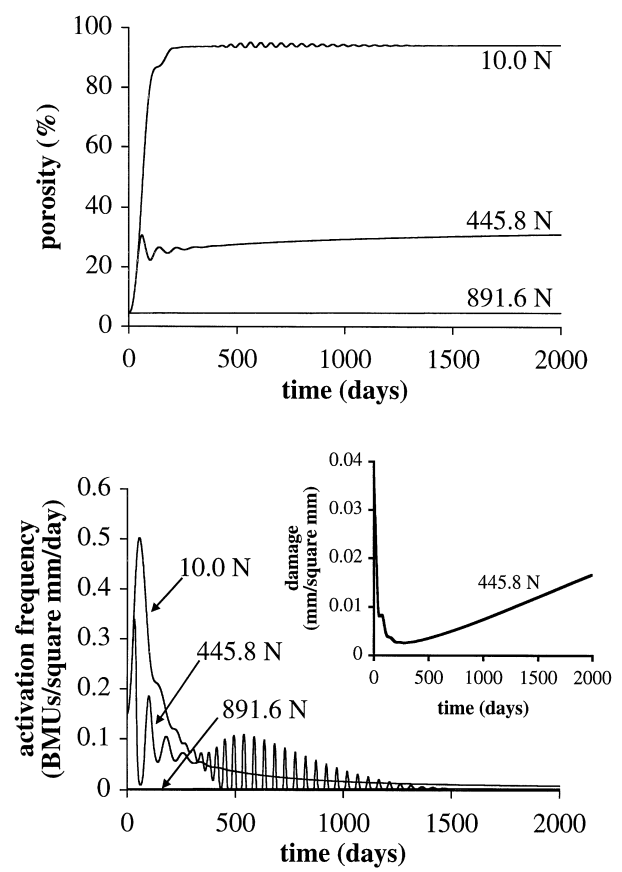

Fig. 4. Porosity (top), BMU activation frequency (bottom), and damage (inset, bottom) responses over time for the model in disuse situations. Porosity of the model increased as it was placed further in disuse. After initial increases, activation frequency returned to near normal conditions. oscillations were greater and lasted longer in the more severe disuse situation. Increasing the maximum BMU activation frequency, $f_{\mathrm{a}(\max )}$, increased the amplitude of the oscillations (Fig. 5). Also, increases in both the amplitudes and periods of these oscillations were observed as the resorption period, $T_{\mathrm{R}}$, was increased (not shown). Changing the reversal and formation periods produced similar but more modest effects. Because remodeling removes damage regardless of how it is activated, there was also a transient reduction in damage during the disuse response (inset, Fig. 4, bottom).

A different kind of instability occurred when the model was sufficiently overloaded. When the applied load was increased to $1400 \mathrm{~N}$ (producing $786 \mu \varepsilon$ initially), and more BMUs were activated to remove the resulting increase in damage, the remodeling space increased, but the system's parameters achieved new steady-state values within 2000 days (Fig. 6). When the applied load was further increased to $1640 \mathrm{~N}(920 \mu \varepsilon$ initially), damage, remodeling rate, and porosity increased as well to accommodate the increased intensity of loading. Then, when the load was increased slightly more, to $1655 \mathrm{~N}$ ( $929 \mu \varepsilon$ initially), the system's behavior entered a new domain where damage, activation frequency, porosity, and strain began to increase rapidly. The amount of loading required to produce such an effect was very sensitive to the exponent $q$, as one would expect (Fig. 7).
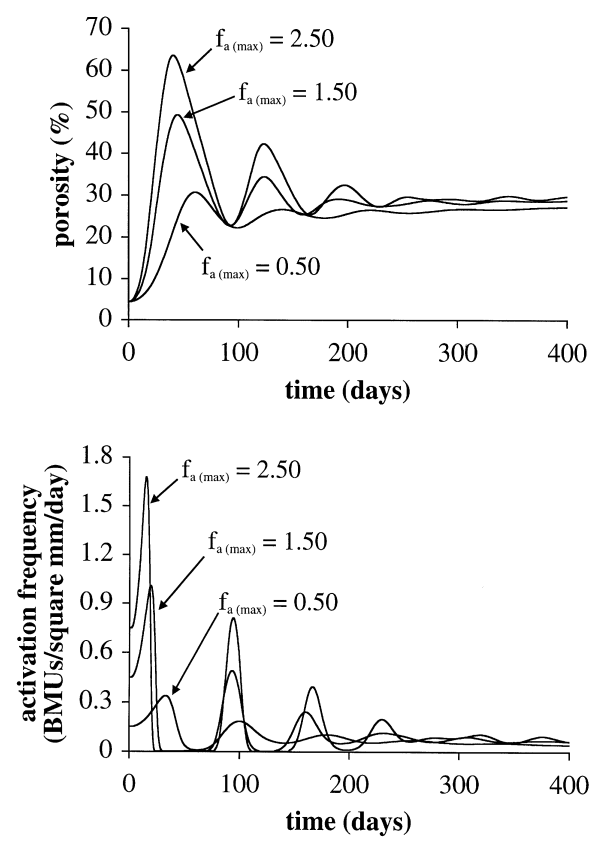

Fig. 5. Increases in the amplitude of the oscillations were observed in disuse situations as the maximum activation frequency $\left(f_{\mathrm{a}}(\max )\right.$ was increased. Shown here are results for $445.8 \mathrm{~N}$. 

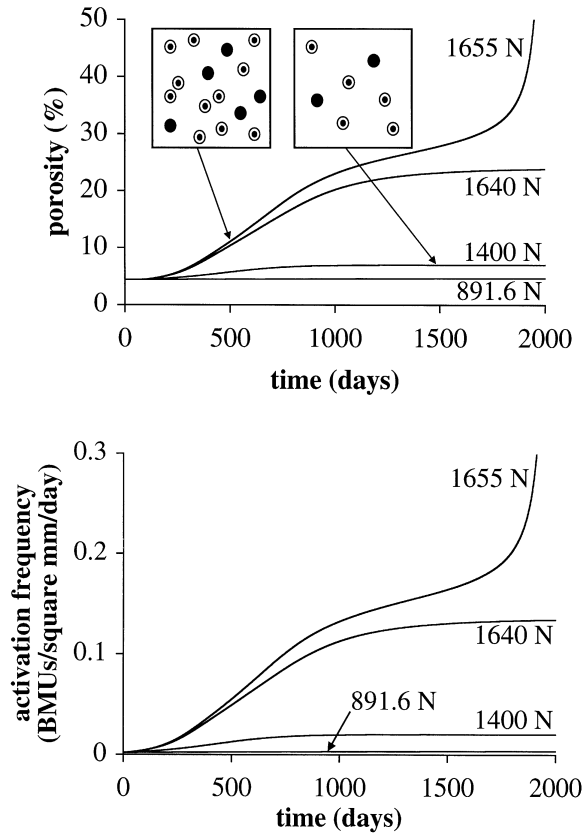

Fig. 6. Porosity and BMU activation frequency responses in the section increased as the load was increased from the equilibrium condition. Below loads of $1640 \mathrm{~N}$, increases in damage formation were offset by corresponding increases in the remodeling rate to remove the damage. Between loads of 1640 and $1655 \mathrm{~N}$, an instability arose as damage formation increased at a rate faster than it could be removed. This happens because strain and damage formation are exponential functions of porosity produced by increased remodeling. In contrast, damage removal is a linear function of the activation frequency (Eq. (8)). Included in the top graph are schematic illustrations of the numbers of active BMUs in a $4 \mathrm{~mm}^{2}$ region of bone. The illustrations compare the active resorbing and refilling BMUs after 500 days of remodeling at a load of $1655 \mathrm{~N}$ (left) with those following 1500 days of remodeling at $1400 \mathrm{~N}$ (right). Resorption cavities are represented by the solid black rings, while refilling BMUs are the rings with black centers. For simplicity, all BMUs are shown to be the same size and their positions in the region were randomly chosen.

The rapid convergence of the numerical results under time increment reduction from 8 to 0.05 days (Figs. 8 and 9) suggests that the simple numerical procedure described here is adequate for the present model. A time step of 1 day was selected for the analysis because it was convenient, and because smaller values resulted in only slight improvements in accuracy.

The behavior of the model was not very sensitive to its initial porosity. In disuse situations, the results were not sensitive to variations in the initial porosity between 0 and $10 \%$ : steady-state porosity values at 2000 days ranged from 94.196 to $94.199 \%$ for a load of $10 \mathrm{~N}$ and 31.07 to $31.16 \%$ at $445.8 \mathrm{~N}$. In overload situations, the results were only sensitive to increases in the initial porosity, due to the relatively large increase in the normalized specific surface area $S_{\mathrm{A}}$. At a load of $1400 \mathrm{~N}$, doubling the initial porosity produced a steady-state porosity approximately twice as large as that in Fig. 6.
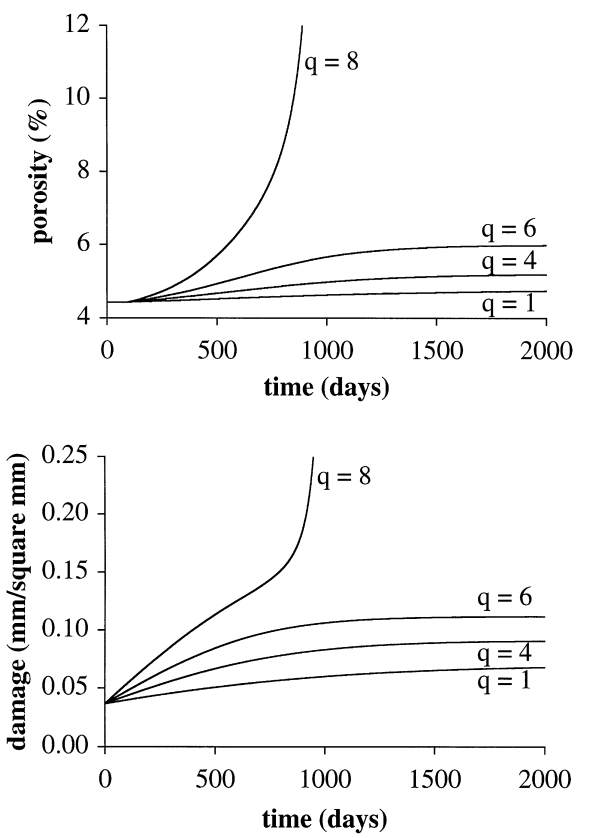

Fig. 7. Effect of varying the exponent $q$ of Eq. (7) on porosity and damage responses. As $q$ was increased from 1 to 8 as the volume element was subjected to a load of $1100 \mathrm{~N}$ (producing $617 \mu \varepsilon$ initially), the capacity of damage formation to exceed damage removal also increased.

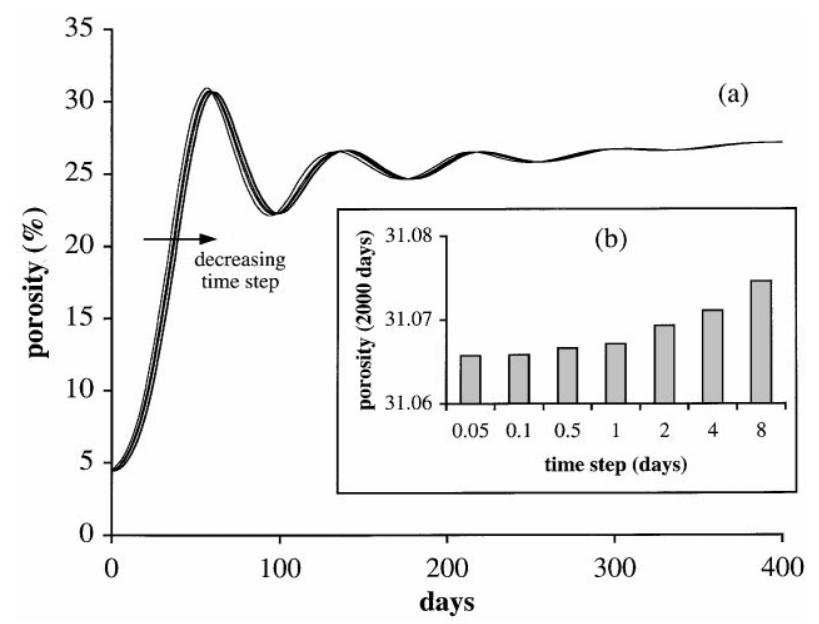

Fig. 8. Porosity responses (a) over the initial 400 days and (b) at 2000 days for a load of $445.8 \mathrm{~N}$ with varying time increments. Time increments of $0.05,0.1,0.5,1,2,4$, and 8 days were examined. All time increments were observed to give stable solutions.

\section{Discussion}

We investigated the dynamic behavior of a new bone remodeling algorithm, in which BMUs are activated in response to either disuse or microdamage. This remodeling algorithm merges concepts from models which simulate the bone remodeling process (Hart and Davy, 1989; Kimmel, 1985; Martin, 1985; Reeve, 1984; Thomsen et al., 1994) with those which simulate the 


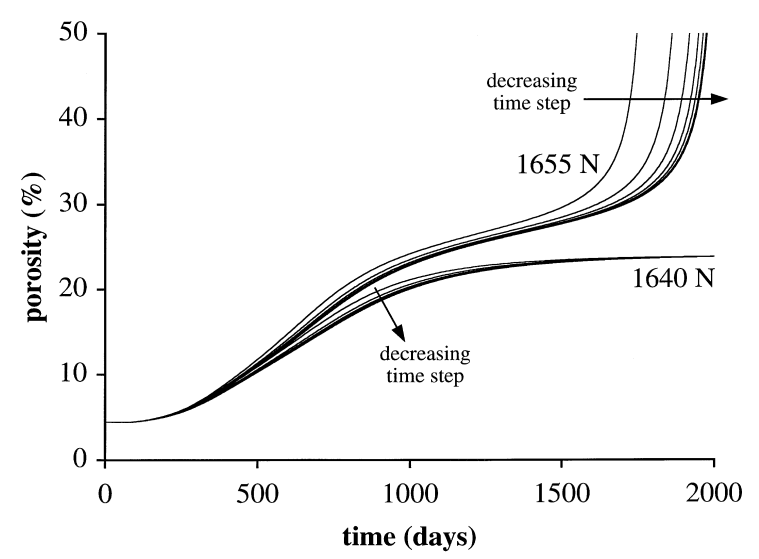

Fig. 9. Effect of varying the time step on the porosity response for loads of 1640 and $1655 \mathrm{~N}$. The behavior was similar for each time step $(0.05,0.1,0.5,1,2,4$, and 8 days) examined in this study.

effects that mechanical variables have on bone density (Beaupré et al., 1990a,b; Carter et al., 1989; Hart et al., 1984; Huiskes et al., 1987; Mullender and Huiskes, 1995; Weinans et al., 1992). Thereby, it attempts to more faithfully reproduce microstructural effects of remodeling. By studying the model's intrinsic responses, we learned that it may manifest distinctly different dynamic behaviors at the extremes of loading: transient oscillations in disuse states and critically sensitive, excessive remodeling in overload situations.

Several investigators have suggested that transient responses may occur during bone adaptation (Frost, 1989; Heaney, 1994; Weinans and Prendergast, 1996; Weinhold et al., 1994). Although most histomorphometric studies are inconclusive regarding this issue, some suggest the existence of transient, oscillatory responses when disuse is imposed (Hardt, 1972; Jaworski et al., 1978; Uhthoff and Jaworski, 1978; Uhthoff et al., 1985). Despite these intriguing results, there are no clear experimental data to support the existence of oscillations similar to those shown in Fig. 4. However, it can also be said that experiments have not been designed to specifically look for them. Since the remodeling variables in the current model can be measured histomorphometrically, the model's results may eventually be validated or refuted by experimental measurements.

The role that the removal of fatigue damage by remodeling plays in preventing stress fractures is of substantial clinical interest. The model presented here predicts that the remodeling system is able to adjust to overloads by increasing the rate of remodeling to cope with increased damage formation, but that this capacity has definite limits. When loaded too much, the model will precipitously enter a "runaway remodeling" mode in which strain, damage, activation frequency, and porosity increase rapidly. Furthermore, this transition is sensitive to small changes in loading or the parameters of the model. This behavior was similar to that described by Martin (1995) and is consistent with the unpredictable clinical course of stress fractures, and with case reports of high turnover in stress fractures (Johnson et al., 1963; Jones et al., 1989)

As with all mathematical models, the present model contains several limiting assumptions. These include the hypothetical "dose-response" functions relating BMU activation frequency to disuse and damage (i.e., Figs. 3a and $b$ ). Variations in the coefficients of these relationships produced slight changes in the steady-state values of the remodeling variables, but the general responses were similar to those described for both disuse and overload. Still, experimental work is required to provide the actual functions. We also need to learn the nature of the fatigue damage that activates remodeling, and the mechanisms by which this occurs. Whatever the nature of this damage, its relationship to various strain tensor components (and other loading conditions) is still unknown.

This brings up another simplifying assumption of the model: using the same mechanical stimulus function to calculate the degree of disuse when strain is low as is used to calculate the local damage. Again, this is an expedient adopted for lack of information about the mechanism by which disuse activates remodeling. The models of Carter and coworkers (Beaupré et al., 1990a,b; Carter et al., 1989; Whalen and Carter, 1988) have established the use of a mechanical stimulus function like that in Eq. (7) as reasonable for defining both disuse and overload, and $q=4$ as a workable exponent. Based on studies of the strain-dependence of bone's fatigue life, values as high as $q=15$ might be considered (Martin, 1992). Our parametric studies show that higher values of $q$ sensitize the model to runaway remodeling in overload situations. Obviously, we need to experimentally determine $q$ under various conditions.

In spite of these limitations, we believe the model is conceptually sound and is a significant step toward modeling what Frost has called bone's "intermediary organization" (Frost, 1983). While incorporating these biological processes adds mathematical complexity, most of the additional variables are histologically measurable. This makes the model amenable to experimental verification. The model also addresses a fundamental inconsistency in the literature of adaptive bone remodeling as it applies to the interiors of bone. Many engineering models of internal bone remodeling assume that bone can be as easily added as subtracted. However, it is difficult to increase bone density by activating remodeling because remodeling cortical bone adds remodeling space and haversian canals, and BMUs in trabecular bone usually remove more bone than they replace, especially in disuse (Minaire et al., 1974). In this view, when bone is overloaded, tissue must be added by periosteal or endosteal bone formation, i.e., "modeling". There is, however, an experimentally 
supported role for increased remodeling when bone is overloaded: the removal of microdamage. The innovation of the present model is that it contains these dual roles for mechanically provoked remodeling: removing bone mass in disuse and removal of damage. Here, we have examined the model's intrinsic dynamic behavior; subsequent applications will incorporate the algorithm into a finite element model of a bony region. In these applications, provision can ultimately be made for the addition or removal of bone on external surfaces by simulated modeling.

\section{Acknowledgements}

This work was supported by NIH grants AR41644 and AR42844. Simulations were run in part on computer facilities provided by a gift from Miss Lorna Talbot to the Veterinary Orthopedic Research Laboratory, University of California, Davis.

\section{References}

Beaupré, G.S., Orr, T.E., Carter, D.R., 1990a. An approach for timedependent bone modeling and remodeling-theoretical development. Journal of Orthopaedic Research 8, 651-661.

Beaupré, G.S., Orr, T.E., Carter, D.R., 1990b. An approach for timedependent bone modeling and remodeling-application: a preliminary remodeling simulation. Journal of Orthopaedic Research 8, 662-670.

Bentolila, V., Boyce, T.M., Fyhrie, D.P., Drumb, R., Skerry, T.M., Schaffler, M.B., 1998. Intracortical remodeling in adult rat long bones after fatigue loading. Bone 23, 275-281.

Birkenhäger-Frenkel, D.H., Birkenhäger, J.C., 1987. Bone appositional rate and percentage of doubly and singly labeled surfaces: comparison of data from 5 and $20 \mu \mathrm{m}$ sections. Bone 8, 7-12.

Brockstedt, H., Bollerslev, J., Melsen, F., Mosekilde, L., 1996. Cortical bone remodeling in autosomal dominant osteopetrosis: a study of two different phenotypes. Bone 18, 67-72.

Brockstedt, H., Kassem, M., Eriksen, E.F., Mosekilde, L., Melsen, F., 1993. Age- and sex-related changes in iliac cortical bone mass and remodeling. Bone 14, 681-691.

Burr, D.B., Martin, R.B., 1993. Calculating the probability that microcracks initiate resorption spaces. Journal of Biomechanics 26, 613-616.

Burr, D.B., Martin, R.B., Schaffler, M.B., Radin, E.L., 1985. Bone remodeling in response to in vivo fatigue microdamage. Journal of Biomechanics 18, 189-200.

Caler, W.E., Carter, D.R., 1989. Bone creep-fatigue damage accumulation. Journal of Biomechanics 22, 625-635.

Carter, D.R., Fyhrie, D.P., Whalen, R.T., 1987. Trabecular bone density and loading history: regulation of connective tissue biology by mechanical energy. Journal of Biomechanics 20, 785-794.

Carter, D.R., Orr, T.E., Fyhrie, D.P., 1989. Relationships between loading history and femoral cancellous bone architecture. Journal of Biomechanics 22, 231-244.

Currey, J., 1984. The Mechanical Adaptations of Bones. Princeton University Press, New Jersey.

Currey, J.D., 1988. The effect of porosity and mineral content on the Young's modulus of elasticity of compact bone. Journal of Biomechanics 21, 131-139.
Eriksen, E.F., 1986. Normal and pathological remodeling of human trabecular bone: three dimensional reconstruction of the remodeling sequence in normals and in metabolic bone disease. Endocrine Reviews 7, 379-408.

Frost, H.M., 1969. Tetracycline-based histological analysis of bone remodeling. Calcified Tissue Research 3, 211-237.

Frost, H.M., 1983. The skeletal intermediary organization. Metabolic Bone Disease and Related Research 4, 281-290.

Frost, H.M., 1989. Transient-steady state phenomena in microdamage physiology: a proposed algorithm for lamellar bone. Calcified Tissue International 44, 367-381.

Frost, H.M., 1998. On rho, a marrow mediator, and estrogen: their roles in bone strength and "mass" in human females, osteopenias, and osteoporoses-insights from a new paradigm. Journal of Bone and Mineral Metabolism 16, 113-123.

Hardt, A.B., 1972. Early metabolic responses of bone to immobilization. Journal of Bone and Joint Surgery 54A, 119-124.

Hart, R.T., Davy, D.T., 1989. Theories of bone modeling and remodeling. In: Cowin, S.C. (Ed.), Bone Mechanics. CRC Press, Boca Raton, FL, pp. 253-277.

Hart, R.T., Davy, D.T., Heiple, K.G., 1984. A computational method for stress analysis of adaptive elastic materials with a view toward applications in strain-induced bone remodeling. Journal of Biomechanical Engineering 106, 342-350.

Heaney, R.P., 1994. The bone-remodeling transient: implications for the interpretation of clinical studies of bone mass change. Journal of Bone and Mineral Research 9, 1515-1523.

Huiskes, R., Weinans, H., Grootenboer, H.J., Dalstra, M., Fudala, B., Slooff, T.J., 1987. Adaptive bone-remodeling theory applied to prosthetic-design analysis. Journal of Biomechanics 20, $1135-1150$.

Jaworski, Z.F.G., Liskova-Kiar, M., Uhthoff, H.K., 1978. Effect of long-term immobilisation on the pattern of bone loss in older dogs. Journal of Bone and Joint Surgery 60B, 420-429.

Johnson, L.C., Stradford, H.T., Geis, R.W., Dineen, J.R., Kerley, E., 1963. Histogenesis of stress fractures. Journal of Bone and Joint Surgery 45A, 1542.

Jones, B.H., Harris, J.M., Vinh, T.N., Rubin, C., 1989. Exerciseinduced stress fractures and stress reactions of bone: epidemiology, etiology, and classification. Exercise and Sport Sciences Reviews 17, 379-422.

Kimmel, D.B., 1985. A computer simulation of the mature skeleton. Bone 6, 369-372.

Lanyon, L.E., Hampson, W.G.J., Goodship, A.E., Shah, J.S., 1975. Bone deformation recorded in vivo from strain gauges attached to the human tibial shaft. Acta Orthopaedica Scandinavica 46, 256-268.

Li, X.J., Jee, W.S.S., Chow, S.-Y., Woodbury, D.M., 1990. Adaptation of cancellous bone to aging and immobilization in the rat: a single photon absorptiometry and histomorphometry study. The Anatomical Record 227, 12-24.

Martin, R.B., 1984. Porosity and specific surface of bone. Critical Reviews in Biomedical Engineering 10, 179-222.

Martin, R.B., 1985. The usefulness of mathematical models for bone remodeling. Yearbook of Physical Anthropology 28, 227-236.

Martin, R.B., 1992. A theory of fatigue damage accumulation and repair in cortical bone. Journal of Orthopaedic Research 10, 818-825.

Martin, R.B., 1995. Mathematical model for repair of fatigue damage and stress fracture in osteonal bone. Journal of Orthopaedic Research 13, 309-316.

Martin, R.B., Burr, D.B., Sharkey, N.A., 1998. Skeletal Tissue Mechanics. Springer, New York.

Mellibovsky, L., Diez, A., Serrano, S., Aubia, J., Pérez-Vila, E., Mariñoso, M.L., Nogués, X., Recker, R.R., 1996. Bone remodeling alterations in myelodysplastic syndrome. Bone 19, 401-405. 
Minaire, P., Meunier, P., Edouard, C., Bernard, J., Courpron, P., Bourret, J., 1974. Quantitative histological data on disuse osteoporosis. Calcified Tissue Research 17, 57-73.

Mori, S., Burr, D.B., 1993. Increased intracortical remodeling following fatigue damage. Bone 14, 103-109.

Mullender, M.G., Huiskes, R., 1995. Proposal for the regulatory mechanism of Wolff's law. Journal of Orthopaedic Research 13, 503-512.

Parfitt, A.M., 1983. The physiologic and clinical significance of bone histomorphometric data. In: Recker, R.R. (Ed.), Bone Histomorphometry: Techniques and Interpretation. CRC Press, Boca Raton, FL, pp. 143-223.

Parfitt, A.M., 1993. Bone age, mineral density, and fatigue damage. Calcified Tissue International 53 (Supplement 1), S82-S86.

Parfitt, A.M., 1994. Osteonal and hemi-osteonal remodeling: the spatial and temporal framework for signal traffic in adult human bone. Journal of Cellular Biochemistry 55, 273-286.

Pirok, D.J., Ramser, J.R., Takahashi, H., Villanueva, A.R., Frost, H.M., 1966. Normal histological, tetracycline and dynamic parameters in human, mineralized bone sections. Henry Ford Hospital Medical Bulletin 14, 195-218.

Prendergast, P.J., Taylor, D., 1994. Prediction of bone adaptation using damage accumulation. Journal of Biomechanics 27, 10671076.

Reeve, J., 1984. A stochastic analysis of iliac trabecular bone dynamics. Clinical Orthopaedics and Related Research 213, 264278.

Rho, J.Y., Ashman, R.B., Turner, C.H., 1993. Young's modulus of trabecular and cortical bone material: ultrasonic and microtensile measurements. Journal of Biomechanics 26, 111-119.

Rubin, C.T., Lanyon, L.E., 1981. Bone remodelling in response to applied dynamic loads. Transactions of the 27th Annual Meeting of the Orthopaedic Research Society 6, 64.

Rubin C.T., Lanyon, L.E., 1983. Regulation of bone mass by peak strain magnitude. Transactions of the 29th Annual Meeting of the Orthopaedic Research Society 8, 70.

Rubin, C.T., Lanyon, L.E., 1984. Regulation of bone formation by applied dynamic loads. Journal of Bone and Joint Surgery 66A, 397-402.

Sarnsethsiri, P., Hitt, O.K., Eyring, E.J., Frost, H.M., 1971. Tetracycline-based study of bone dynamics in pycnodysostosis. Clinical Orthopaedics and Related Research 74, 301-312.
Schaffler, M.B., Choi, K., Milgrom, C., 1995. Aging and matrix microdamage accumulation in human compact bone. Bone 17 , $521-525$.

Schaffler, M.B., Li, X.J., 1990. Immobilization induced bone loss: quantitative histological studies of cortical bone resorption. Transactions of the 36th Annual Meeting of the Orthopaedic Research Society 15, 187.

Steiniche, T., Christiansen, P., Vesterby, A., Hasling, C., Ullerup, R., Mosekilde, L., Melsen, F., 1994. Marked changes in iliac crest bone structure in postmenopausal osteoporotic patients without any signs of disturbed bone remodeling or balance. Bone 15, 73-79.

Stout, S.D., Lueck, R., 1995. Bone remodeling rates and skeletal maturation in three archaeological skeletal populations. American Journal of Physical Anthropology 98, 161-171.

Thomsen, J.S., Mosekilde, Li., Boyce, W., Mosekilde, E., 1994. Stochastic simulation of vertebral trabecular bone remodeling. Bone 15, 655-666.

Turner, C.H., Anne, V., Pidaparti, R.M.V., 1997. A uniform strain criterion for trabecular bone adaptation: do continuum-level strain gradients drive adaptation? Journal of Biomechanics 30, 555-563.

Uhthoff, H.K., Jaworski, Z.F.G., 1978. Bone loss in response to longterm immobilisation. Journal of Bone and Joint Surgery 60B, 420 429.

Uhthoff, H.K., Sékaly, G., Jaworski, Z.F.G., 1985. Effect of long-term nontraumatic immobilization on metaphyseal spongiosa in young adult and old beagle dogs. Clinical Orthopaedics and Related Research 192, 278-283.

Underwood, E.E., 1970. Quantitative Stereology. Addison-Wesley Publishing Co., Reading, MA.

Weinans, H., Huiskes, R., Grootenboer, H.J., 1992. Effects of material properties of femoral hip components on bone remodeling. Journal of Orthopaedic Research 10, 845-853.

Weinans, H., Prendergast, P.J., 1996. Tissue adaptation as a dynamical process far from equilibrium. Bone 19, 143-149.

Weinhold, P.S., Gilbert, J.A., Woodard, J.C., 1994. The significance of transient changes in trabecular bone remodeling activation. Bone $15,577-584$.

Whalen, R.T., Carter, D.R., 1988. Influence of physical activity on the regulation of bone density. Journal of Biomechanics 21, 825-837. 